\title{
Introducing a medical ambulatory care service
}

\author{
Author: Shianne Varrier
}

\section{Aims}

To set up a safe and direct in-hours medical service for patients not requiring an overnight admission to be promptly seen and discharged on the same day, thereby reducing 0 -day admissions and streamlining access to acute medicine.

\section{Methods}

A 2-week pilot of the ambulatory care unit (ACU) service was run in December 2017 during working hours, 8am-4pm. The service was provided by a dedicated medical specialty registrar (StR) and overseen by an acute medicine consultant.

The ACU StR triaged all general practitioner (GP) referrals. Patients were booked in for admission to ACU on the day or later in the week and seen on admission directly, then promptly reviewed by the acute medical physician on call within 2-3 hours of attendance. Management plans were made either requiring admission, discharge back to GP or further attendance postinvestigation.

\section{Results}

The number of calls attended by the ACU middle grade varied between $12-20$ a day.

> Approximately one-quarter (25\%) of calls required over the phone advice to the referring GP and therefore potentially avoided attendance.

> Approximately four patients were seen by within the ACU department on the day with $<25 \%$ conversion rate to admission. Most were either discharged directly or re-attended later in the week.

The number of calls from GPs to the acute medical StR fell significantly in-hours, allowing them to concentrate on other activities. Feedback demonstrated greater satisfaction with the management of the acute medical take.

The number of medical overnight admissions/prolonged length of stay over the day (8am to $8 \mathrm{pm}$ ) on-call shift dropped by approximately $15-20 \%$.

All patients admitted to ACU were seen within 30 minutes of arrival and a management plan was put in place within 2-3 hours.

Author: Royal College of Physicians chief registrar, Royal Surrey County Hospital, Surrey, UK

\section{Conclusion}

A permanent ACU service within the medical division was approved.

The service is run by a dedicated junior doctor between 12-8pm (identified as the busiest period for the on-call medical team), increasing junior doctors' acute medical take numbers by $25 \%$ and allowing GP referrals to be diverted still from the on-call team. The ACU service has been given dedicated space within the hospital, which will allow for ACU 'hot clinic' slots to be set up within an appropriate setting, overseen by the acute medicine consultants to allow appropriate follow up and arrangement of more complex patients. This will help to achieve trust compliance with the new Shape of Training internal medicine model for future medical trainees from 2019 onward.

\section{Conflict of interest statement}

None. 